\title{
Are the Consequences of Gastrointestinal Infections of SARS-CoV-2 Underestimated?
}

\author{
Kai Hilpert* \\ Institute of Infection and Immunology, St. George's University of London (SGUL), London, UK \\ ${ }^{\star}$ Corresponding author: Kai Hilpert, Institute of Infection and Immunology, St. George’s University of London (SGUL), London, UK; E-mail: khilpert@sgul.ac.uk
}

Received: August 07, 2020; Accepted: August 11, 2020; Published: August 18, 2020

With more than 7.8 million confirmed cases and over 430,000 deaths COVID-19 has had an impact on the whole world (16.06.2020). In many countries the peak of the first wave seems to be passing and strict lockdown measures are being eased gradually. It is important to implement risk mitigation procedures to ensure the safety of those people returning to the workplace and participating in "public life". Despite mounting evidence that faeces are infectious there seems to be little discussion of this, no public awareness of risk, or implementation of potential mitigation strategies. In addition, lasting alterations in the gut microbiome of patients infected with SARS-CoV-2 have been observed in at least two pilot studies. Dysbiosis in the gut microbiome influences different biochemical functions and is associated with diverse diseases, effecting the immune system, metabolic states and mental health. In consequence, there could be unidentified long-term health risks of SARS-CoV-2 infections in the gut.

In a meta-analysis of 60 studies, comprising 4243 COVID-19 patients Cheung et al. showed that the pooled prevalence of any gastrointestinal symptoms was $17.6 \%$ (95\% CI: $12.3-24.5 \%)$, with $11.8 \%$ occurring with non-severe COVID 19 symptoms and $17.1 \%$ with severe symptoms [1]. Not all studies reported all individual gastrointestinal symptoms, consequently the pooled prevalence of anorexia was $26.8 \%$ (95\% CI: $16.2-40.8$ ), diarrhoea $12.5 \%$ (95\% CI: 9.6-16.0), nausea/vomiting 10.2\% (95\% CI: 6.6-15.3), and abdominal pain/discomfort 9.2\% (95\% CI: 5.7-14.5).

Using immunofluorescent staining, Xiao et al. showed expression of the ACE2 protein in the glandular cells of gastric, duodenal, and rectal epithelia [2]. They also demonstrated staining of viral nucleocapsid protein in the same epithelia cell populations. In addition, the authors showed that of 73 hospitalized patients in the study, 53\% tested positive for SARS-CoV-2 RNA in stool. Wu et al. confirmed these findings and went a step further and followed the patients over time showing that respiratory samples remained positive for SARS-CoV-2 RNA for a mean of 16.7 days (SD 6.7) and faecal samples remained positive for a mean of 27.9 days (SD 10.7) after first symptom onset [3]. These findings have been repeated and verified in a number of additional studies, in adults and children, asymptomatic, mild and severe COVID-19 patients. In addition, Wu et al. showed that from 60 patients that were released from hospital after clinical recovery $10 \%$ were still positive for SARS-CoV-2 RNA in anal swab [4]. At least four independent studies have been published reporting the isolation of infectious SARS-CoV-2 from stool samples. For example, Wang et al. reported the isolation of live SARS-
CoV-2 from stool samples (non-diarrhoea) from two patients [5]. This is of particular interest since various studies have shown the production of bioaerosols during toilet flushing [6]. That is significant since new data shows that airborne transmission plays a major role for the spread of SARS-CoV-2 [7]. Indeed, in a report Liu et al. studied the potential aerosol transmission of SARS-CoV-2 in two Wuhan hospitals [8]. The authors reported elevated levels of viral RNA in the patient toilet areas and recommended proper disinfection and ventilation. In addition, Van Doremalen et al. showed that viable SARS-CoV-2 could be detected in aerosols up to 3 hours after aerosolization, and up to 2-3 days on plastic and stainless steel [9].

First studies of the gut microbiome of COVID-19 patients showed a significant decrease in diversity and abundance compared to healthy control (HC) [10]. This was combined with the increased relative abundance of opportunistic pathogens like Streptococcus and Rothia. The study showed significant disease-specific shifts in the overall microbiota composition between COVID-19 and H1N1 patients and HC. Changes in the gut microbiome have been shown to associate with various diseases, for example IBS, IBD, type 2 diabetes, cardiovascular condition and depression. Further studies are needed to evaluate the long term effects on SARS-CoV-2 infections in the gut and possibly a restorative treatment regime needs to be developed.

Taken together these results strongly indicate that SARS-CoV-2 infections in the gut have short- and long-term effects. The shortterm effects are gastrointestinal symptoms and in about $50 \%$ of the patients viral shedding in stool occurs. Toilet flushing can create infectious bioaerosols that are a potential risk factor for oral/nasalfaecal transmission of SARS-CoV-2. Cleaning procedures of toilets in hospitals, care facilities, schools, nurseries, work places, restaurants, trains, busses, airplanes and public spaces will need to be looked at and perhaps improved.

\section{Conflict of Interest}

I am a founder, director and shareholder of TiKa Diagnostics Ldt., however the company is specialized in diagnostics of Mycobacteria (for example TB) in humans and animals, and has not provided funding or any other support/influence for this review.

\section{Acknowledgement}

I thank Martin Cranage, Mark Bodman-Smith, Deborah Baines, Kathleen Kirmer and Ralf Mikut for their feedback. 


\section{References}

1. Cheung KS, Hung IF, Chan PP, Lung KC, Tso E, et al. (2020) Gastrointestinal Manifestations of SARS-CoV-2 Infection and Virus Load in Fecal Samples from the Hong Kong Cohort and Systematic Review and Meta-analysis. Gastroenterology 159: 81-95. [crossref]

2. Xiao F, Tang M, Zheng X, Liu Y, Li X, et al. (2020) Evidence for gastrointestinal infection of SARS-CoV-2. Gastroenterology 158: 1831-1833. [crossref]

3. Wu Y, Guo C, Tang L, Hong Z, Zhou J, et al. (2020b) Prolonged presence of SARSCoV-2 viral RNA in faecal samples. Lancet Gastroenterol Hepatol 5: 434-435. [crossref]

4. Wu J, Liu X, Liu J, Liao H, Long S, et al. (2020a) Coronavirus Disease 2019 Test Results After Clinical Recovery and Hospital Discharge Among Patients in China. JAMA Netw 3: e209759. [crossref]

5. Wang W, Xu Y, Gao R, Lu R, Han K, et al. (2020) Detection of SARS-CoV-2 in Different Types of Clinical Specimens. JAMA - J. Am. Med 323: 1843-1844. [crossref]
6. Johnson DL, Mead KR, Lynch RA, Hirst DVL (2013) Lifting the lid on toilet plume aerosol: A literature review with suggestions for future research. Am J Infec Control 41: 254-258. [crossref]

7. Zhang R, Li Y, Zhang AL, Wang Y, Molina MJ (2020) Identifying airborne transmission as the dominant route for the spread of COVID-19. Proc Natl Acad Sci U S A 117: 14857-14863. [crossref]

8. Liu Y, Ning Z, Chen Y, Guo M, Liu Y, et al. (2020) Aerodynamic analysis of SARSCoV-2 in two Wuhan hospitals. Nature 582: 557-560. [crossref]

9. van Doremalen N, Lloyd-Smith JO, Munster VJ, Bushmaker T, Morris DH, et al. (2020) Aerosol and surface stability of HCoV-19 (SARS-CoV-2) compared to SARSCoV-1 456 Full author list: Neeltje van Doremalen. medRxiv.

10. Zuo T, Zhang F, Lui GCY, Yeoh YK, Li AYL, et al. (2020) Alterations in Gut Microbiota of Patients With COVID-19 During Time of Hospitalization. Gastroenterology doi: 10.1053/j.gastro.2020.05.048. [crossref]

\section{Citation:}

Hilpert K (2020) Are the Consequences of Gastrointestinal Infections of SARS-CoV-2 Underestimated? Infect Dis Ther Volume 1(2): 1-2. 